\title{
Correction to: Land Cover Classification and Accuracy Evaluation Based on Object- Oriented Spatial Features of GF-2
}

Xiaomao Chen, Jiakun Li, and Yuanfa Ji

\section{Correction to:}

Chapter "Land Cover Classification and Accuracy Evaluation Based on Object-Oriented Spatial Features of GF-2" in:

H. Gao et al. (Eds.): Communications and Networking, LNICST 352, https://doi.org/10.1007/978-3-030-67720-6_21

The original version of this chapter was revised: The name of Yuanfa Li has been corrected to Yuanfa Ji. 